Discussion Paper No. 492

\title{
DESIGNING GHG EMISSIONS \\ TRADING INSTITUTIONS \\ IN THE KYOTO PROTOCOL: \\ AN EXPERIMENTAL APPROACH
}

\author{
Yoichi Hizen \\ and \\ Tatsuyoshi Saijo
}

January 2000

The Institute of Social and Economic Research

Osaka University

6-1 Mihogaoka, Ibaraki, Osaka 567-0047, Japan 


\title{
Designing GHG Emissions Trading Institutions in the Kyoto Protocol: An Experimental Approach\#
}

\author{
Yoichi Hizen ${ }^{1} 2$ \\ and \\ Tatsuyoshi Saijo ${ }^{3,4}$
}

December 1998

\# This research was partially supported by CREST (Core Research for Evolutional Science and
Technology) of the Japan Science and Technology Corporation (JST) and Grant in Aid for Research
1278, 08453001 and 0873003 of the Ministry of Education, Science and Culture in Japan. We also
thank Professors Peter Bohm, James C. Cox, Timothy N. Cason, R. Mark Isaac, Michael McAleer,
Charles R. Plott and participants of the CREST workshop for helpful comments and discussions, and
Dr. Yasuko Kawashima of the National Institute for Environmental Studies in Japan for providing
marginal abatement cost information.
${ }^{1}$ Graduate School of Economics, Osaka University, Toyonaka, Osaka 560-0043, Japan
${ }^{2}$ Research Fellow of the Japan Society for the Promotion of Science.
${ }^{3}$ Institute of Social and Economic Research, Osaka University, Ibaraki, Osaka 567-0047, Japan
${ }^{4}$ CREST, Japan Science and Technology Corporation, 3-12-40 Hiroo, Shibuya, Tokyo 150-0012, Japan. 


\begin{abstract}
We re-evaluate two experiments by Hizen and Saijo $(1999 a, b)$ to examine the performance of bilateral trading and double auction institutions in GHG emissions trading. It is found that: (i) the efficiency of both institutions is quite high, regardless of the disclosure or closure of contracted price and/or marginal abatement cost curve information; (ii) marginal abatement costs are equalized over time in both institutions; (iii) contracted prices roughly converge to the competitive price over time in a double auction, but not quite so in bilateral trading.
\end{abstract}

Journal of Economic Literature Classification Numbers: C92, Q31, Q38

\title{
Correspondence:
}

Tatsuyoshi Saijo

Institute of Social and Economic Research

Osaka University

Ibaraki, Osaka 567-0047

Japan

Phone 01181-6 (country \& area codes)

6879-8582 (office)/ 6878-2766 (fax)

E-mail: saijo@iser.osaka-u.ac.jp 


\section{Introduction}

The Kyoto Protocol to the Climate Convention in December 1997 calls for Annex B countries (that is, advanced countries and some countries that are in transition to market economies) to reduce their average greenhouse gas (GHG) emissions over 2008-2012 to about five percent below 1990 levels. In order to implement this goal, it authorizes three major mechanisms called the Kyoto mechanism. These are emissions trading, joint implementation and the Clean Development Mechanism. However, we must design the details of these mechanisms as almost no details are given in the Protocol. The Conference of Parties at Buenos Aires in November 1998 following the Kyoto Conference adopted a "Plan of Action," but this plan is a timetable regarding what should be argued when, and hence no details of the protocol are given. The focus of this paper is to design desirable institutions for GHG emissions trading through the use of experimental economics.

Several candidates for emissions trading institutions have been proposed, such as auctions, bilateral trading, and a mixture of these two. However, no scientific bases have been given to support them. For example, as a supplemental institution to auctions, some countries have proposed bilateral trading. A typical response to this proposal by economists, among others, is twofold: (i) it would be inefficient in the sense that some dissatisfied traders could not be eliminated due to mismatching; and (ii) the revelation of contracted prices would improve its efficiency. These views are not based upon either economic theory or empirical evidence. ${ }^{1}$ As a matter of fact, economic theory often assumes that the market is a black box, and does not look into the details of trading institutions. Moreover, actual data on real economic activities cannot inform us how

\footnotetext{
${ }^{1}$ Feldman (1973) showed that allocations through bilateral trading can attain Pareto efficiency in a finite number of steps.
} 
efficient the trading institution is. For example, most of the trades in sulfur dioxide allowance trading in the U.S.A. have been carried out through bilateral trading. Some have been undertaken through an annual auction designed by the Environmental Protection Agency (EPA), but it is hard to tell how efficient it is since no comparisons have been made with other institutions. ${ }^{2}$

What would be the best way to evaluate several alternative institutions without having data and with only limited experience? A new method of understanding the performance of alternatives has emerged in economics, namely, experimental economics. For example, the EPA designed a new auction method for sulfur dioxide allowance trading, hoping to realize relatively high allowance prices. However, the actual prices around the beginning of trading were significantly lower than those expected by specialists. Cason (1995) and Cason and Plott (1996) established laboratory experiments where the trading institution was exactly the same as the EPA auction. Participants (called subjects) in the experiments were motivated by monetary rewards. That is, subjects who performed well in the laboratory EPA auction received greater compensation than those who did not perform well. What they found was striking. The experimental price data were well below the market price, which is exactly the opposite of the EPA's expectation!

In survey articles, Muller and Mestelman (1998) and Godby, Mestelman and Muller (1988) provide many new findings on emissions trading experiments. Among others, experimental economists have found that: (i) allowing banking permits over time smoothes contracted prices across time periods, and (ii) a trader who has some market power other than in the emissions trading market can influence the emissions trading

\footnotetext{
${ }^{2}$ Some evaluation of sulfur dioxide emissions trading has just started. See, for example, Schmalensee et al. (1998), Stavins (1998), and Joskow et al. (1998).
} 
market, and hence the introduction of the emissions market reduces the efficiency of the whole economy.

In this paper, we re-examine two papers by Hizen and Saijo $(1999 a, b)$ who focused on bilateral trading and double auction institutions with a special feature called the dual role property. In most previous experiments in emissions trading, each subject is assigned to be a buyer or a seller, but not both. That is, once a subject is a buyer, she cannot sell her allowances even when the price is extremely high. In these experiments, every subject can be a buyer and a seller depending on the prices, so that a subject can play dual roles in emissions trading. The initiator of this dual role property in emissions trading is Bohm (1997). He reported bilateral trading experiments among four teams consisting of experienced public officials or experts appointed by the Energy Ministries. Each team represented one of the four Nordic countries, and has some information on the marginal abatement cost curves of all teams. The asks and bids were exchanged by fax without revealing this information to other teams. It took four days to complete this experiment. The resulting prices were very close to the competitive equilibrium price and the efficiency of the allocation was $97 \%$, which is surprisingly high.

Following Bohm's important experiment, Hizen and Saijo (1999a) designed a bilateral trading experiment with two controls: (i) disclosure or closure of contracted prices, and (ii) disclosure or closure of marginal abatement cost curves. Thus, there are four different treatments. In this way, we can understand which case would be the most appropriate or efficient way to reduce GHG emissions. Furthermore, Hizen and Saijo (1999b) conducted a double auction experiment with the same setting as the bilateral trading experiment and then compared two institutions. In the double auction, both buyers and sellers can actively post and accept prices in a public manner. In Hizen and 
Saijo (1999b), the control was the disclosure or closure of marginal abatement cost curves since the contracted price information was revealed to all subjects in the double auction.

The first finding is that the efficiency of both institutions is very high, regardless of information closure or disclosure. That is, disclosure of contracted prices and/or marginal abatement cost information does not improve efficiency. Allocation efficiency was more than $99 \%$ in 6 sessions, and $98 \%$ and $92 \%$ in the remaining sessions of the bilateral trading experiment, and it was more than $99 \%$ in all five sessions in the double auction experiment. This finding contrasts strikingly with the widely accepted view on bilateral trading. Second, marginal abatement costs are equalized in almost all sessions in both institutions. Third, while the contracted prices did not converge to the competitive equilibrium price in the bilateral trading experiment, they roughly converged to the price in the double auction experiment. This observation is different from Bohm's. Fourth, subjects who could exercise market power did not use that power in the bilateral trading experiment, but subjects who had market power in the double auction experiment earned greater profits than they could have earned at the competitive equilibrium price. In each session, we had six subjects who were supposed to represent Russia, Ukraine, U.S.A., Poland, EU, and Japan. In our setting, only the U.S.A. had market power, and the subjects who were assigned this role could reduce their quantity demanded in order to lower the price.

The paper is organized as follows. Section 2 investigates bilateral trading and section 3 examine a double auction. In section 4, we discuss why efficiency is so high, consider the implication of disclosure of contracted prices, and compare the two institutions. Section 5 discusses the future agenda. 


\section{Bilateral Trading}

\subsection{Experimental Design}

The bilateral trading experiment has two controls: (i) disclosure or closure of contracted prices, and (ii) disclosure or closure of marginal abatement cost curves. Therefore, there are four treatments. Repeating the same treatment twice yields eight sessions. In what follows, "O" represents "disclosure" and "X" represents "closure". For example, "OX2" indicates session 2 in the price disclosure, marginal cost curve closure treatment.

We recruited at least six students for each session by campus-wide advertisement at Osaka University during October 1998. These students were told that there would be an opportunity to earn money in a research experiment. None of them had prior experience in a bilateral trading experiment. It took approximately 160 minutes for each session. The mean payoff per subject was $\$ 31.25$ ( $\$ 1=115$ yen). The maximum payoff was $\$ 66.09$, and the minimum payoff was $\$ 17.39$.

Figure 1 is around here

Let us describe an "OO" session. Subjects were seated at desks in a relatively large room and listened to a tape-recorded voice giving instructions. In this part, each subject received a sample graph (see Figure 1). The upper half is a sample marginal abatement cost curve. Each subject was told that the initial position is at $0 .{ }^{3}$ If the subject moves to the right, she buys the allowance and earns benefits, and if she moves to the left, she sells the allowance and obtains a profit. All possible situations were depicted in the lower half

\footnotetext{
${ }^{3}$ We implicitly presume that position 0 is the position where each country attains the goal required by the Kyoto Protocol. In other words, the experimental setting asks what kind of trading should be done in order to achieve that goal. That is, we did not address the non-compliance issue.
} 
of Figure 1. After receiving instructions on how to transact with other subjects, all subjects took an examination to check their understanding of the instructions. The best six subjects continued the experiment, and the rest were asked to leave the room with $\$ 13$. Then each subject was assigned an identification number from 1 to 6 and, at the same time, each subject received her own marginal abatement cost curve. In the "OO" session, each subject received Figure 2 with all six abatement cost curves, and had fifteen minutes to examine it. ${ }^{4}$ Then bilateral trading started. Since every subject had a tag with an identification number, subjects could identify other subjects' marginal abatement cost curves. Each subject could move around the room freely to find a subject with whom to transact. During negotiations, subjects were not allowed to talk. Only numbers (price and quantity), and "yes" and "no" symbols on their negotiation sheets, were exchanged in order to avoid information leakage. Once a pair reached agreement, they reported the price and quantity to an experimenter, who announced these numbers on a blackboard (i.e., contracted price disclosure). The maximum time for negotiations was 60 minutes, and subjects could end their negotiations early if all subjects agreed to it. The full 60 minutes was used in all sessions except "XO2."

Figure 2 is around here

During the experiment, we did not use any country names or the term "emissions trading." That is, subjects faced a situation where trading of an abstract commodity was conducted with an abstract price.

\subsection{Experimental Results}

Economists care about the market (or competitive) equilibrium price where the demand and supply curves intersect since the sum of benefit and profit of all subjects is

\footnotetext{
${ }^{4}$ Since information on the marginal abatement cost curves around 2010 is hard to obtain, we draw these
} 
maximized at this price. Keeping this in mind, we define the efficiency of bilateral trading as follows:

Sum of benefit and profit of each subject

Sum of benefit and profit at market equilibrium

That is, the maximum efficiency should be one. In our design, the competitive equilibrium price ranges from 118 to 120 so we regard 119, which is the midpoint between 118 and 120, as the competitive equilibrium price. At this price, the total amount of benefit and profit (that is, the maximum amount that these six subjects can enjoy) is 6990 . In Table 1, the top row indicates the name of the sessions, the left column shows the I.D. numbers of subjects, and the numbers in parentheses are their benefits or profits at the competitive equilibrium price. In each cell, the upper figure is the actual benefit or profit that the subject earned, and the lower figure is the efficiency of this subject. For example, the 0.732 figure for subject 1 in the "OO2" session is the ratio between 1870 and 2555, which we call individual efficiency.

\begin{tabular}{|c|r|r|r|r|r|r|r|r|}
\hline & OO1 & OO2 & OX1 & OX2 & XO1 & XO2 & XX1 & XX2 \\
Subject No. & & & & & & & & \\
\hline $1(2555)$ & 1420 & 1870 & 960 & 1710 & 1510 & 1100 & 1460 & 1600 \\
(Russia) & 0.556 & 0.732 & 0.376 & 0.669 & 0.591 & 0.431 & 0.571 & 0.626 \\
\hline $2(1290)$ & 1140 & 914 & 360 & 1665 & 1320 & 940 & 1536 & 2370 \\
(Ukraine) & 0.884 & 0.709 & 0.279 & 1.291 & 1.023 & 0.729 & 1.191 & 1.837 \\
\hline $3(610)$ & 685 & 683 & 2060 & 372 & 1846 & 615 & 583 & 550 \\
(U.S.A.) & 1.123 & 1.120 & 3.377 & 0.610 & 3.026 & 1.008 & 0.956 & 0.902 \\
\hline $4(390)$ & 520 & 570 & 850 & 530 & 500 & 555 & 910 & 500 \\
(Poland) & 1.333 & 1.462 & 2.179 & 1.359 & 1.282 & 1.423 & 2.333 & 1.282 \\
\hline $5(620)$ & 800 & 1105 & 1300 & 755 & -150 & 1080 & 81 & 150 \\
(EU) & 1.290 & 1.782 & 2.097 & 1.218 & -0.242 & 1.742 & 0.131 & 0.242 \\
\hline $6(1525)$ & 2425 & 1800 & 1450 & 1844 & 1400 & 2700 & 2390 & 1800 \\
(Japan) & 1.590 & 1.180 & 0.951 & 1.209 & 0.918 & 1.770 & 1.567 & 1.180 \\
\hline Sum(6990) & 6990 & 6942 & 6980 & 6876 & 6426 & 6990 & 6960 & 6970 \\
& 1 & 0.993 & 0.999 & 0.984 & 0.919 & 1 & 0.996 & 0.997 \\
\hline
\end{tabular}

Table 1. Efficiency of Bilateral Trading

curves based upon some information provided by Dr. Kawashima. 
As Table 1 shows, the efficiency of each session is quite high, except for "XO1." The reason for the low efficiency level in this session is that subject 5 traded with other subjects even though she suffered a loss. Individual efficiencies are quite different even for the same subject number. Statistical tests show that Russia consistently earned a much lower profit than that of the competitive equilibrium and Poland earned a greater profit than that of the competitive equilibrium. The efficiency of the other countries is close to one. As we discuss later, the only country that had some market power was the U.S.A.

Figure 3 is around here

Figure 3 shows efficiency changes over time. After sixteen minutes, the efficiency of allocation in all sessions except for session "XX2," was greater than $80 \%$, and it was more than $90 \%$ in 6 sessions of 8 after 25 minutes. Efficiency usually is monotonically increasing, but in session "OX2" efficiency moved up and down. This was due to the fact that one subject bought allowances at a loss, but sold it at a relatively high price. Summarizing these findings, we have:

Observation 1. (i) The efficiency of bilateral trading is almost one, regardless of closure or disclosure of price and marginal abatement cost information.

(ii) Russia's efficiency is low, Poland's efficiency is high, and the efficiency of the other countries is close to one.

(iii) The efficiency of allocation in 6 sessions of 8 was more than $90 \%$ after 25 minutes.

Figures 4-1 and 4-2 show contracted prices and quantities, together with asks and bids, over time. The gray horizontal line in each small graph shows the competitive equilibrium price range of 118-120. The left-hand side number of a square is the seller's 
subject number, the right-hand side number is the buyer's subject number, and the number under the square is the contracted quantity. If the variance of the last three contracted prices is significantly smaller than the variance of the first three prices, then we say that the contracted price sequence converges. We find convergence of contracted prices in five of eight sessions, but no information disclosure effect is observed.

Figures 4-1 and 4-2 are around here

Observation 2. (i) Contracted average prices in session "XX" (closure of prices and closure of marginal abatement cost curves) roughly equal the competitive equilibrium price, but the variances of prices in sessions XX1 and XX2 are larger than those of the rest.

(ii) Average prices cannot be said to equal the competitive equilibrium price in sessions other than the "XX" sessions.

(iii) The average price of the last three contracts is not equal to the competitive equilibrium price in every session.

(iv) The convergence of contracted prices is found in five of eight sessions, but no information disclosure effect on convergence is observed.

By the nature of bilateral trading, the price contracted by a pair of subjects is determined by negotiation. Even though several other contracted prices have already been announced, a pair of subjects cannot reach an agreement if either of them rejects these prices. For this reason, the competitive equilibrium price cannot play the role of the standard of trade.

The effect of disclosure of contracted prices can be calculated by comparing the "OO" and "XO" sessions, and the "OX" and "XX" sessions. Since the variances of 
contracted prices in sessions "OX1" and "OX2" cannot be said to be the same, we omitted these sessions from the comparison. Under this constraint, we compare the "OO" sessions with the "XO" sessions. No differences are observed among the variances of these sessions. For the same reason mentioned in the above paragraph, we compare the "XO" sessions with the "XX" sessions to measure the effect of disclosure of marginal abatement cost curve information. We find that the variance of contracted prices in the "XO" sessions is smaller than in the "XX" sessions. Summarizing these facts, we have:

Observation 3. (i) Assuming that each subject knows other subjects' marginal abatement cost curves well, the disclosure of contracted prices does not have any impact on the variance of contracted prices.

(ii) Under the closure of contracted prices, the disclosure of marginal abatement cost curves reduces the variance of contracted prices.

Behind efficiency, we can see how marginal abatement costs changed over time. Due to the step-function nature of our marginal abatement cost curve, we must be careful when evaluating marginal costs. For example, the marginal abatement cost of Russia in session "OO1" was 90 after 25 minutes. Checking the raw data, we find that Russia sold exactly 55 units of emissions allowance in 25 minutes. Therefore, if the subject wanted to sell one more unit, its marginal abatement cost would have been 120 (see Figure 2). Taking account of this fact, we have:

Observation 4. Except for the EU subject in session "XO1," the marginal abatement costs of all subjects approach the competitive equilibrium price, but the contracted prices do not. 
In order to understand how much market power a country has, we need an aggregate excess demand curve of all subjects regarding marginal abatement cost curves as the excess demand curves for emissions allowances. After careful examination, we find that the only country that has market power in our design is the U.S.A. Table 1 shows that the benefits of the U.S.A. were more than three times the benefit at the competitive equilibrium in two sessions of eight. A statistical test shows that the U.S.A. did not exercise market power in any session. Most probably, the subjects could not exploit the marginal abatement cost curve information to use such market power.

\section{Double Auction}

\subsection{Experimental Design}

In a double auction, all proposals, including contracted prices and quantities, are disclosed. Therefore, our double auction experiment has only one control, namely disclosure or closure of marginal abatement cost curves. We had three disclosure sessions and two closure sessions, and hence five sessions in total. Following the previous section, we denote these sessions by "O3", "X2", and so on. The only difference between our double auction and bilateral trading is in the trading method. In the double auction, an auctioneer calls on a subject who raises her hand the earliest. Then the subject provides her I.D. number, sell or buy, the quantity and the price per unit. For example, the subject says, "Subject five sells ten units at one hundred dollars per unit." The auctioneer projects the proposal on an OHP screen. After the proposal, a subject who raises her hand the earliest can trade with the proposer. The accepted quantity is smaller than or equal to the proposed quantity. We impose the "improvement rule" on proposals in our double auction, that is, asks (bids) must be successively lower (higher). 
In our double auction experiment, the mean payoff per subject was $\$ 28.20$, the maximum payoff was $\$ 44.70$, and the minimum payoff was $\$ 17.39$. The maximum time for an auction was 60 minutes, and subjects could end the auction early if all subjects agreed to it. The full 60 minutes was used in three of five sessions.

\subsection{Experimental Results}

As Table 2 shows, the efficiency of each session is quite high. Statistical tests show that Poland consistently earned a lower profit than that of the competitive equilibrium and the U.S.A. earned a greater profit than that of the competitive equilibrium. The efficiency of the other countries is close to one.

\begin{tabular}{|c|r|r|r|r|r|}
\hline & $\mathrm{O} 1$ & $\mathrm{O} 2$ & $\mathrm{O} 3$ & $\mathrm{X1}$ & $\mathrm{X} 2$ \\
\hline Subject No. & & & & & \\
\hline 1 (2555) & 2410 & 2410 & 1981 & 2260 & 2865 \\
(Russia) & 0.943 & 0.943 & 0.775 & 0.885 & 1.121 \\
\hline 2 (1290) & 1320 & 1320 & 520 & 1770 & 1120 \\
(Ukraine) & 1.023 & 1.023 & 0.403 & 1.372 & 0.868 \\
\hline 3 (610) & 850 & 865 & 1144 & 681 & 1270 \\
(U.S.A.) & 1.393 & 1.418 & 1.875 & 1.116 & 2.082 \\
\hline 4 (390) & 200 & 350 & 230 & 209 & 355 \\
(Poland) & 0.513 & 0.897 & 0.590 & 0.536 & 0.910 \\
\hline $5(620)$ & 750 & 500 & 1380 & 700 & 0 \\
(EU) & 1.210 & 0.806 & 2.226 & 1.129 & 0.000 \\
\hline 6 (1525) & 1430 & 1515 & 1695 & 1350 & 1360 \\
(Japan) & 0.938 & 0.993 & 1.111 & 0.885 & 0.892 \\
\hline Sum (6990) & 6960 & 6960 & 6950 & 6970 & 6970 \\
& 0.996 & 0.996 & 0.994 & 0.997 & 0.997 \\
\hline
\end{tabular}

Table 2. Efficiency of the Double Auction

Figure 5 shows efficiency changes over time. After seventeen minutes, the efficiency of allocation in all sessions except for session "X2," was greater than $70 \%$, and was more than $90 \%$ in all sessions after 44 minutes. Efficiency usually is monotonically increasing, but in sessions "X1" and "X2" efficiency reached $100 \%$ once and then fell slightly. For example, in session "X2," one subject sold allowances at a loss, expecting that 
she would buy them at a lower price later but could not. Such a loss was not observed when the marginal abatement cost information was disclosed.

Figure 5 is around here

Observation 5. (i) The efficiency of the double auction is almost one, regardless of closure or disclosure of marginal abatement cost information.

(ii) Poland's efficiency is low, the U.S.A.'s efficiency is high, and the efficiency of the other countries is close to one.

(iii) The efficiency of allocation in all sessions is more than 90\% after 44 minutes.

Figure 6 is around here

Figure 6 shows contracted prices and quantities, together with asks and bids, over time. We examine the average price of all contracts and that of the last three contracts to see where the prices converge. We have:

Observation 6. (i) Regardless of closure or disclosure of marginal abatement cost information, convergence of prices is observed.

(ii) Average prices of all contracts in sessions "O1", "O2" and "X1" equal the competitive equilibrium price.

(iii) Average prices of the last three contracts in sessions "O1", "X1" and "X2" equal the competitive equilibrium price. 
We examine the effect of disclosure of marginal abatement cost curves. The variances of contracted prices in the "O" sessions are smaller than those in the " $\mathrm{X}$ " sessions. In competitive equilibrium, Russia, Ukraine and Poland are sellers while the U.S.A., EU and Japan are buyers. In the "O" sessions, Russia, Ukraine and Poland only sold the allowances, while the U.S.A., EU and Japan only bought them. The numbers of trade in sessions "O1", "O2" and "O3" were 9, 8 and 7, respectively, while those in sessions "X1" and "X2" were 11 and 12, respectively.

Observation 7. The disclosure of marginal abatement cost curves:

(i) reduces the variance of contracted prices;

(ii) makes Russia, Ukraine and Poland only sell, and the U.S.A., EU and Japan only buy;

(iii) reduces the number of trades.

Behind efficiency, we can see how marginal abatement costs changed over time.

Observation 8. Marginal abatement costs of all subjects approach the competitive equilibrium price.

In our double auction experiment, marginal abatement costs converged less rapidly than in bilateral trading. We conjecture that this arises because, in the double auction, at most one pair can trade at the same time while at most three pairs can do so in bilateral trading.

\section{Discussions and Comparisons}


Extremely high efficiency in Observations 1 and 5 is partly due to the fact that each country can buy and sell the allowance depending on the price, which we call the dual role property of a trader. We consider that the property is essential in emissions trading. Consider the following simple demand and supply curves with two suppliers and two buyers. Each supplier has just one unit of a good. On the other hand, each buyer wants to buy just one unit of the good. Apparently the competitive equilibrium price must be between 4 and 7 , and the social surplus becomes 8 . If buyers can only buy and sellers can only sell, a mismatch can easily occur. Consider a pair in which the buyer's value is 10 and the seller's cost is 7. If they trade with each other, the surplus is 3. Consider another pair in which the buyer's value is 4 and the seller's cost is 2 . The surplus of this pair is 2 and hence the sum of the surplus is 5 , which is less than 8 . After these trades, no mutually beneficial trade will occur. Now consider the case in which everyone can buy and sell. After these trades, the buyer whose value is 4 has one unit of the good and hence can sell it. Since the seller whose cost is 7 does not have any good, she can buy it. If this pair trades with each other, they can enjoy 3 units of surplus, so that the total surplus becomes 8. Actually, starting from any trading pair, we can find a path that leads to a Pareto efficient allocation.

Figure 7 is around here

In our bilateral trading experiment, every session had at least one subject who was a buyer and a seller in the same session. Apparently, this dual role property helped to improve the efficiency of allocation in all sessions. On the other hand, in the double auction experiment, no sessions with marginal abatement cost curve disclosure had such a subject, but every session with its closure had at least one such subject. In the double 
auction with the marginal abatement cost curve disclosure, every subject understood who should be a buyer and a seller from Figure 2, and hence no dual role trader was generated during the auction. However, the dual role property was important to improve efficiency in the double auction under the marginal abatement cost closure.

Most of the previous emissions trading experiments in the literature has not taken account of the dual role property. For example, Cason and Gangadharan (1998) reported that efficiencies are quite low in an experiment where subjects publicly post proposed terms of trade through an Electronic Bulletin Board that captures the Regional Clean Air Incentives Market (RECLAIM) in the Los Angeles area. Cason and Gangadharan partly attribute this low efficiency to some transaction costs due to the Bulletin Board system. We would conjecture that the dual role property may drastically improve its efficiency. In both bilateral trading and double auction experiments, the variances of weighted contracted prices around the beginning of transactions are quite large, but toward the end the variances of weighted contracted prices in double auction sessions are sufficiently smaller than those in bilateral trading sessions. Furthermore, we find that contracted prices around the end of transactions in double auction sessions are much closer to the competitive equilibrium price than those in bilateral trading sessions. In these respects, the double auction is better than bilateral trading.

As for the effect of marginal abatement cost disclosure, the variances of weighted contracted prices with information disclosure are smaller than those with information closure in double auction sessions. In bilateral trading, the former are smaller than the latter if the contracted prices are not disclosed. The disclosure of contracted price information in bilateral trading does not affect the variances of the contracted prices if the abatement cost information is revealed. Since the number of sessions in our experiments 
is limited, we cannot conclude some definite effects. However, it seems that the information of marginal abatement costs of other countries may reduce some variances of contracted prices in both bilateral trading and the double auction.

The experiments did not show any significant effects of disclosure of contracted prices on the variance in bilateral trading. This does not necessarily imply that the closure of contracted prices is better than disclosure. First, if the disclosure or closure of contracted prices does not matter, there is no reason why they should be hidden. Although the experimental results did not show any significant difference, something unexpected might occur in future emissions trading. For this safety reason, transactions should be undertaken in public. Second, the transaction of GHG emissions allowances is not a truly private economic activity. As it would affect absolutely everyone, it should be done in public.

As Figures 4-1, 4-2, and 6 show, the number of asks and bids in bilateral trading is larger than the number in the double auction. The average number in bilateral trading is about four times as large as that in the double auction. If we think that this number is an indicator of economic transaction costs, we might conclude that the double auction is better than bilateral trading. However, this statement belies the fact that a large volume of asks and bids can be transmitted through a computer network such as the internet at only a nominal cost.

Subjects who played the U.S.A. role in the double auction experiment obtained a greater profit than they could have earned at the competitive equilibrium price. This does not necessarily indicate that the subjects exercised market power. We conjecture that subjects in the double auction may have much broader information on the market than the subjects in bilateral trading since bids and asks are public in the double auction. This fact 
might trigger the U.S.A. subjects to use their market power. Clearly, further studies are needed to understand this market power issue.

\section{Concluding Remarks}

In these experiments, it was found that, first, the efficiency of both bilateral trading and double auction is quite high. This high efficiency may come from the dual role property of traders. Second, marginal abatement costs are equalized over time in both trading institutions. Third, contracted prices did not converge to the competitive price over time in bilateral trading, but they converged relatively to the competitive price in the double auction. Fourth, subjects who had market power did not use it in bilateral trading, but subjects who had market power in the double auction experiment obtained greater profits than at the competitive price.

If a policy goal is only to achieve cost effectiveness, either institution can attain such a goal. On the other hand, if a policy maker cares about "equity," in the sense that the same unit of allowance must be traded with the same price, the double auction is better than bilateral trading. Furthermore, if one thinks that some transaction costs increase as the number of asks and bids increases, the double auction is better than bilateral trading.

Our comparison of two institutions is a prelude to several more experiments that aim at designing the entire emissions trading institutions. In order to provide a solid basis for such a design, we plan to conduct the following: (1) Banking experiment, (2) Noncompliance experiment, (3) Liability experiment and (4) Market power experiment. 


\section{REFERENCES}

Bohm, Peter, A Joint Implementation as Emission Quota Trade: An Experiment Among Four Nordic Countries, Nord 1997:4 by Nordic Council of Ministers, June, 1997.

Cason, Timothy N., "An Experimental Investigation of the Seller Incentives in the EPA's Emission Trading Auction," American Economic Review 85(4), September 1995, pp. 905-22.

Cason, Timothy N. and Charles R. Plott, "EPA's New Emissions Trading Mechanism: A Laboratory Evaluation," Journal of Environmental Economics and Management 30(2), March 1996, pp. 133-60.

Cason, Timothy N. and Lata Gangadharan, "An Experimental Study of Electronic Bulletin Board Trading for Emission Permits," Journal of Regulatory Economics 14, 1998, pp. 5573.

Feldman, Allan M., "Bilateral Trading, Processes, Pairwise Optimality, and Pareto Optimality," Review of Economic Studies 40(4), October 1973, pp. 463-73.

Godby, Robert W., Stuart Mestelman, and R. Andrew Muller, "Experimental Tests of Market Power in Emission Trading Markets," in Environmental Regulation and Market Structure, Emmanuel Petrakis, Eftichios Sartzetakis, and Anastasios Xepapadeas (Eds.), Cheltenham, United Kingdom: Edward Elgar Publishing Limited, forthcoming, September, 1998.

Hizen, Y., and T. Saijo, "Price Disclosure, Marginal Abatement Cost Information and Market Power in a Bilateral GHG Emissions Experiment," unpublished manuscript, ISER, Osaka University, 1999a.

Hizen, Y., and T. Saijo, "Comparing Bilateral Trading and Double Auction Institutions in GHG Emissions: Experimental Results," unpublished manuscript, ISER, Osaka University, $1999 b$.

Joskow, Paul L. Richard Schmalensee, and Elizabeth M. Bailey, "The Market for Sulfur Dioxide Emissions," American Economic Review 88(4), September 1998, pp.669-685. 
Muller, R. Andrew and Stuart Mestelman, "What Have We Learned From Emissions Trading Experiments," mimeo., forthcoming in Managerial and Decision Economics, July, 1998.

Stavins, Robert N., "What Can We Learn from the Grand Policy Experiment? Lessons from $\mathrm{SO}_{2}$ Allowance Trading," Journal of Economic Perspectives 12(3), Summer, 1998, pp.69-88.

Schmalensee, Richard, Paul L. Joskow, A. Denny Ellerman, Juan Pablo Montero, and Elizabeth M. Bailey, "An Interim Evaluation of Sulfur Dioxide Emissions Trading," Journal of Economic Perspectives 12(3), Summer, 1998, pp.53-68. 


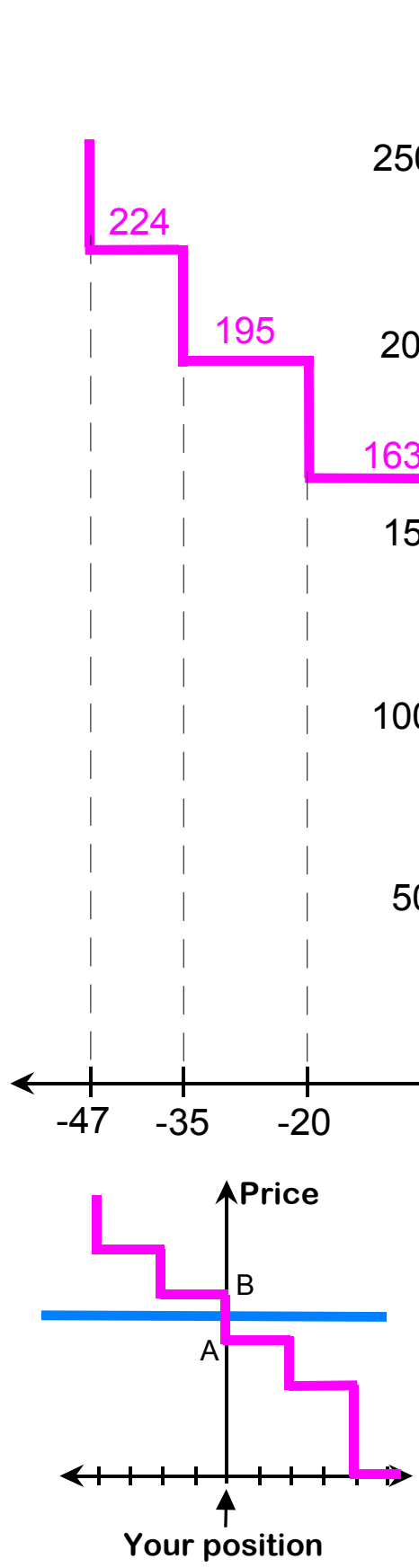

No buy or no sell

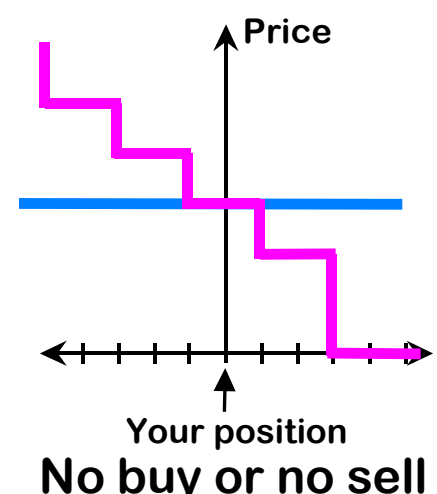

No buy or no sell

Price

This is a sample graph. We will distribute the graph that you will use after the instruction.

120

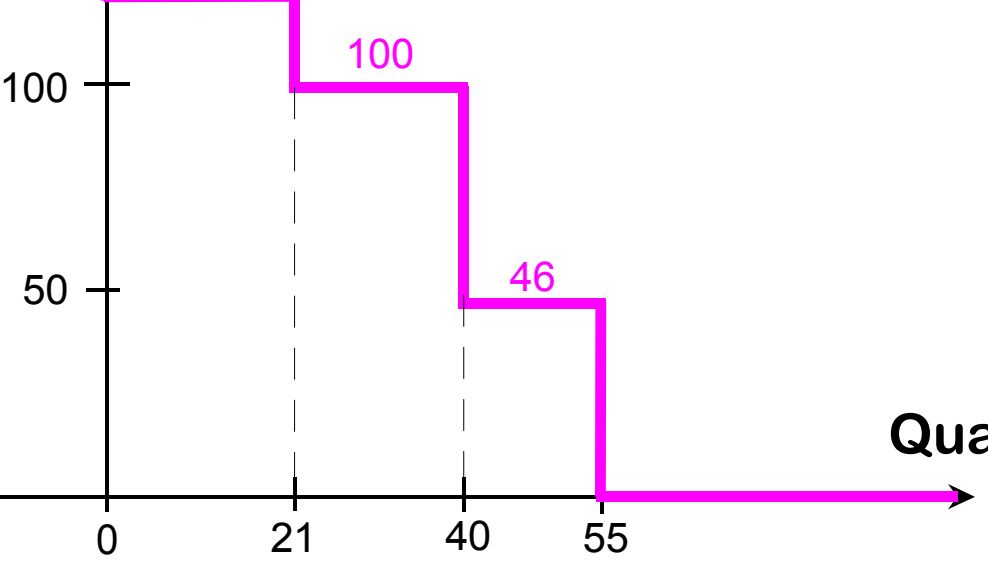

Quantity

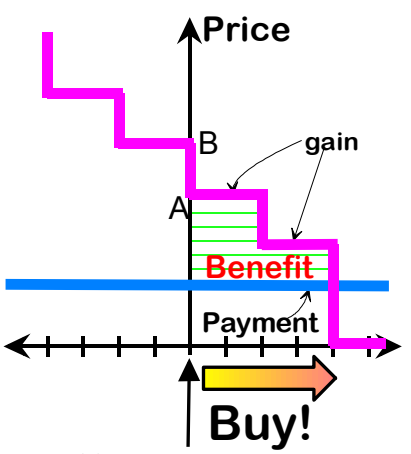

Your position

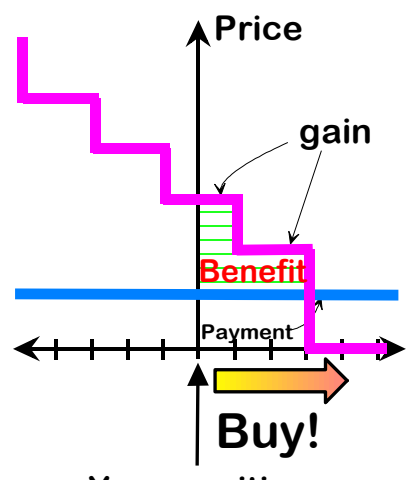

Your position

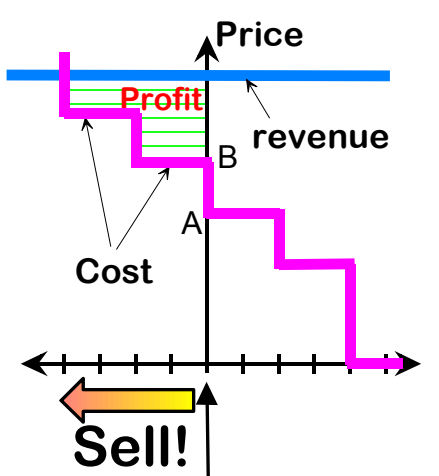

Your position

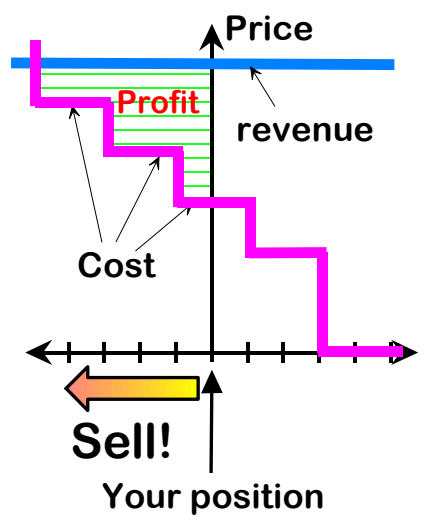

Figure 1. A Sample Graph 


\section{1-Russia 2-Ukraine 3-USA 4-Poland 5-EU 6-Japan}

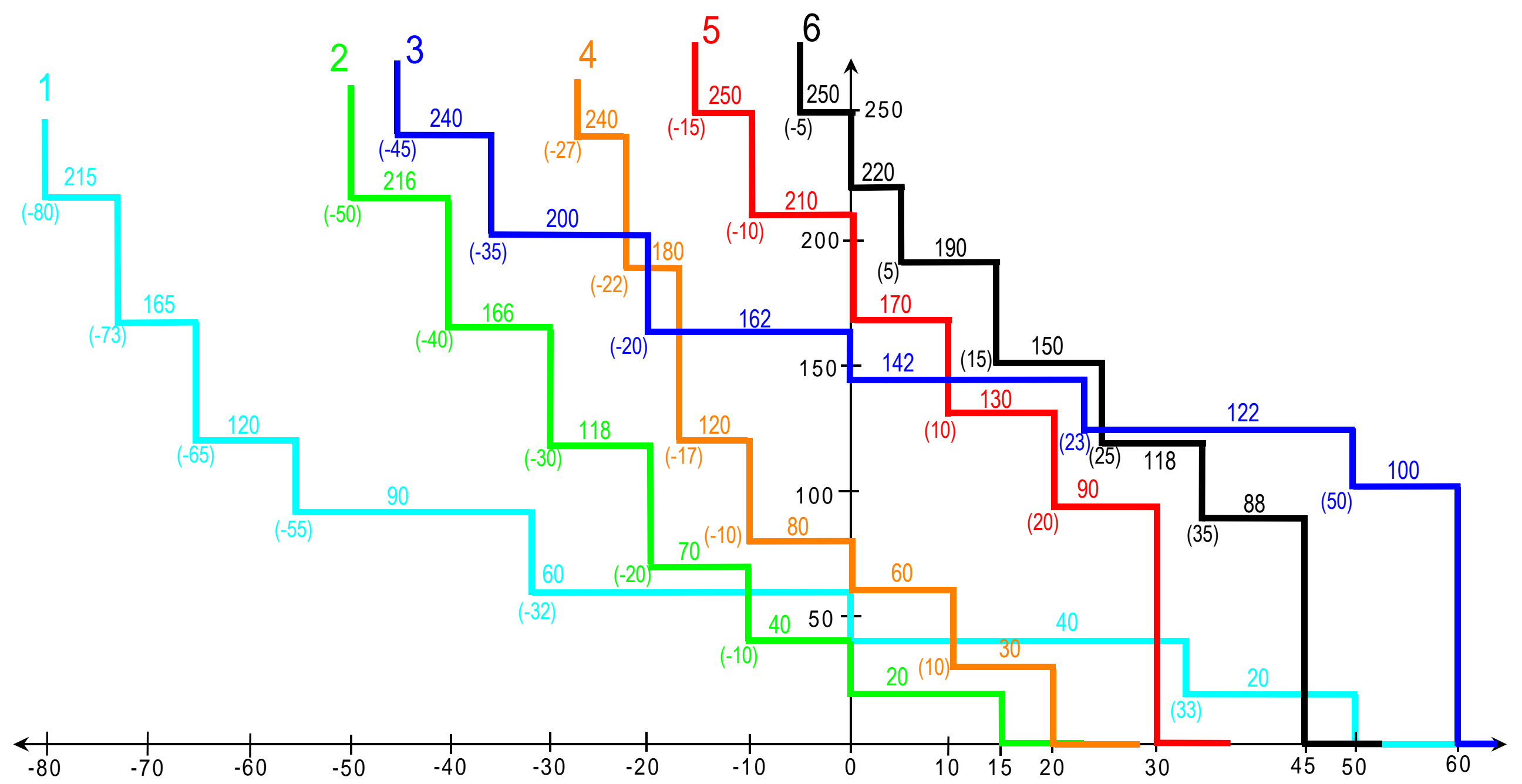

No country names were given to subjects in the experiment.

Figure 2. All Marginal Abatement Cost Curves 


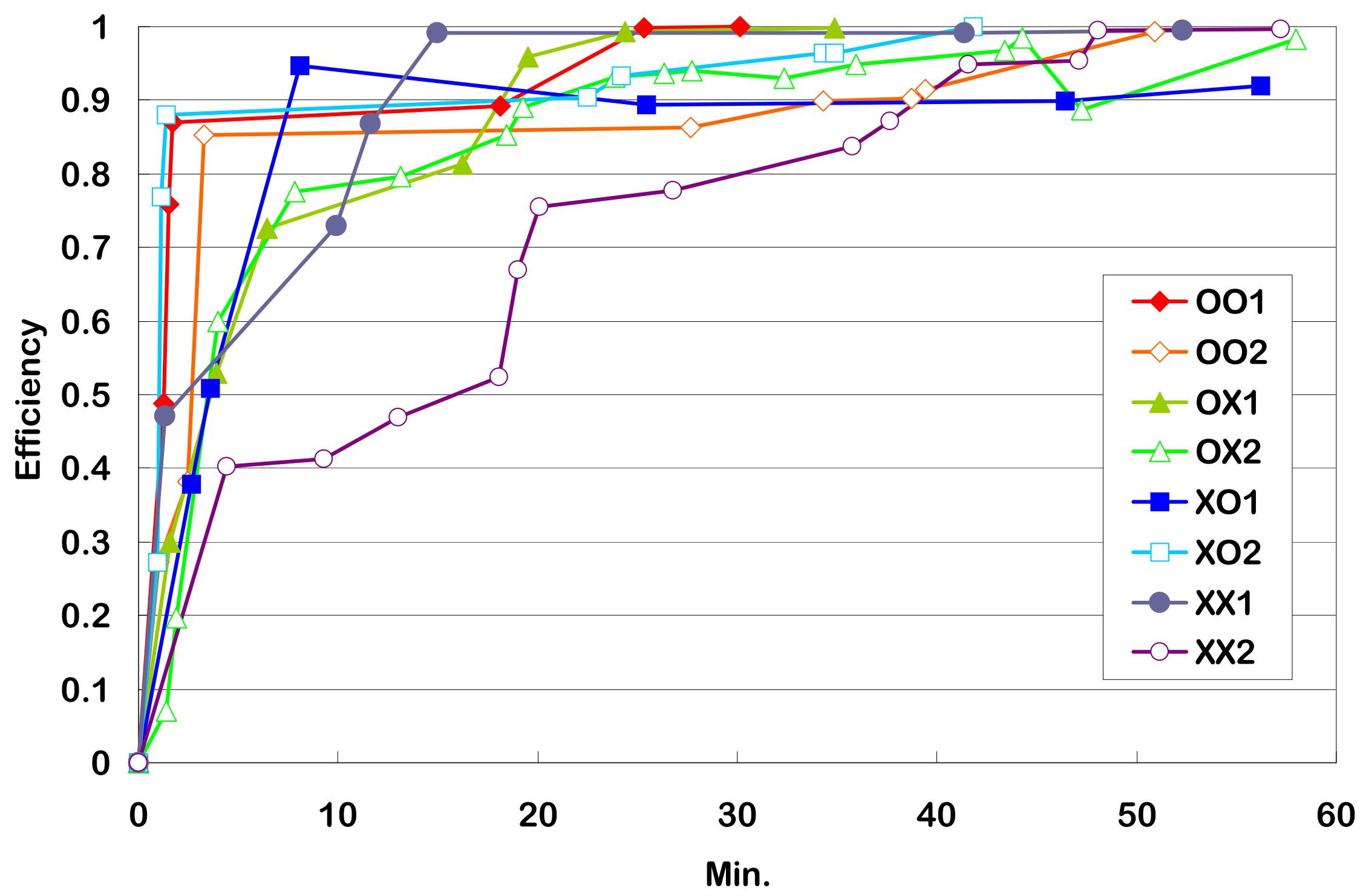

Figure 3. Efficiency over Time in Bilateral Trading 

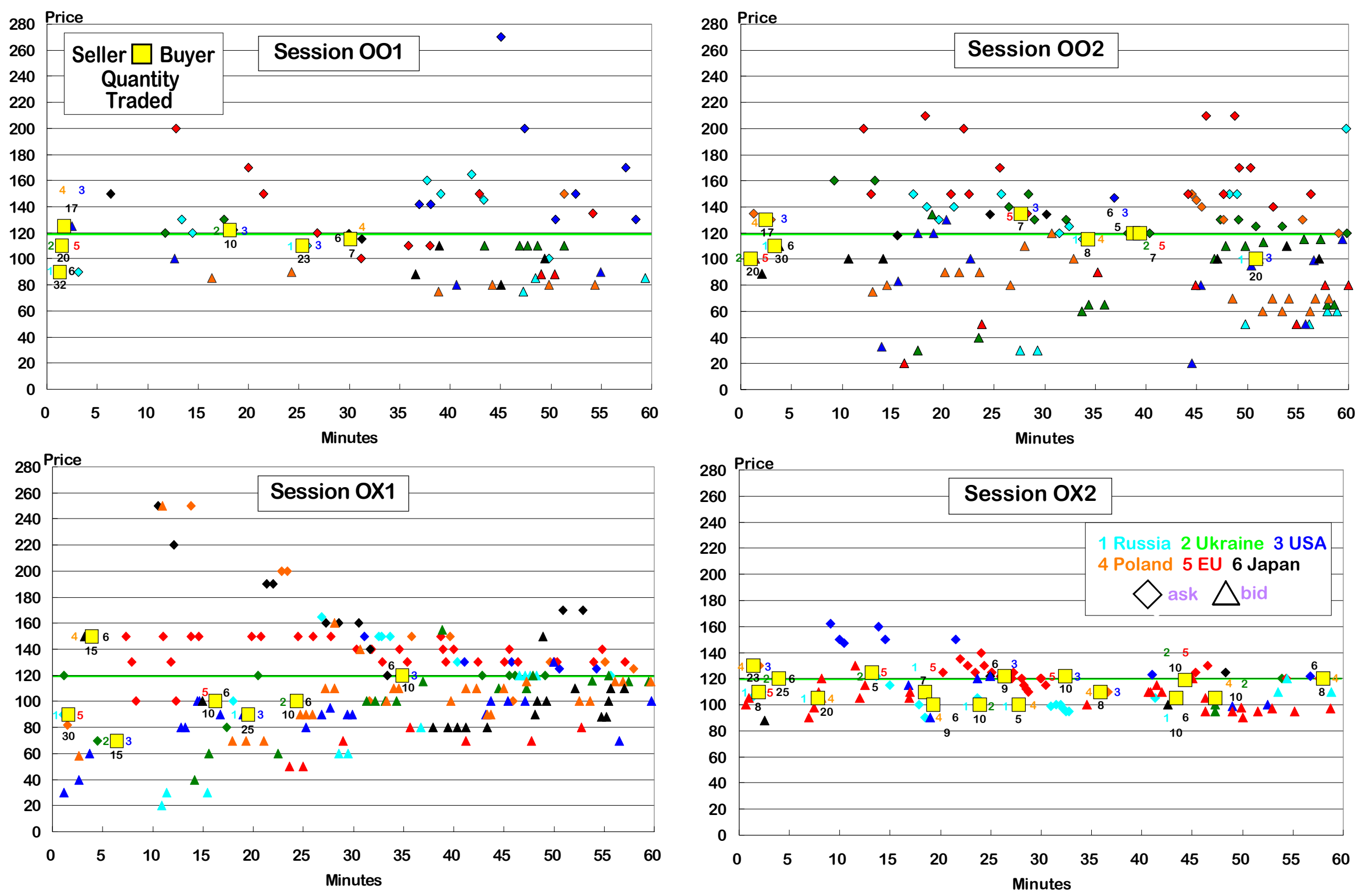

Figure 4-1. Contracts, Asks and Bids in Bilateral Trading 

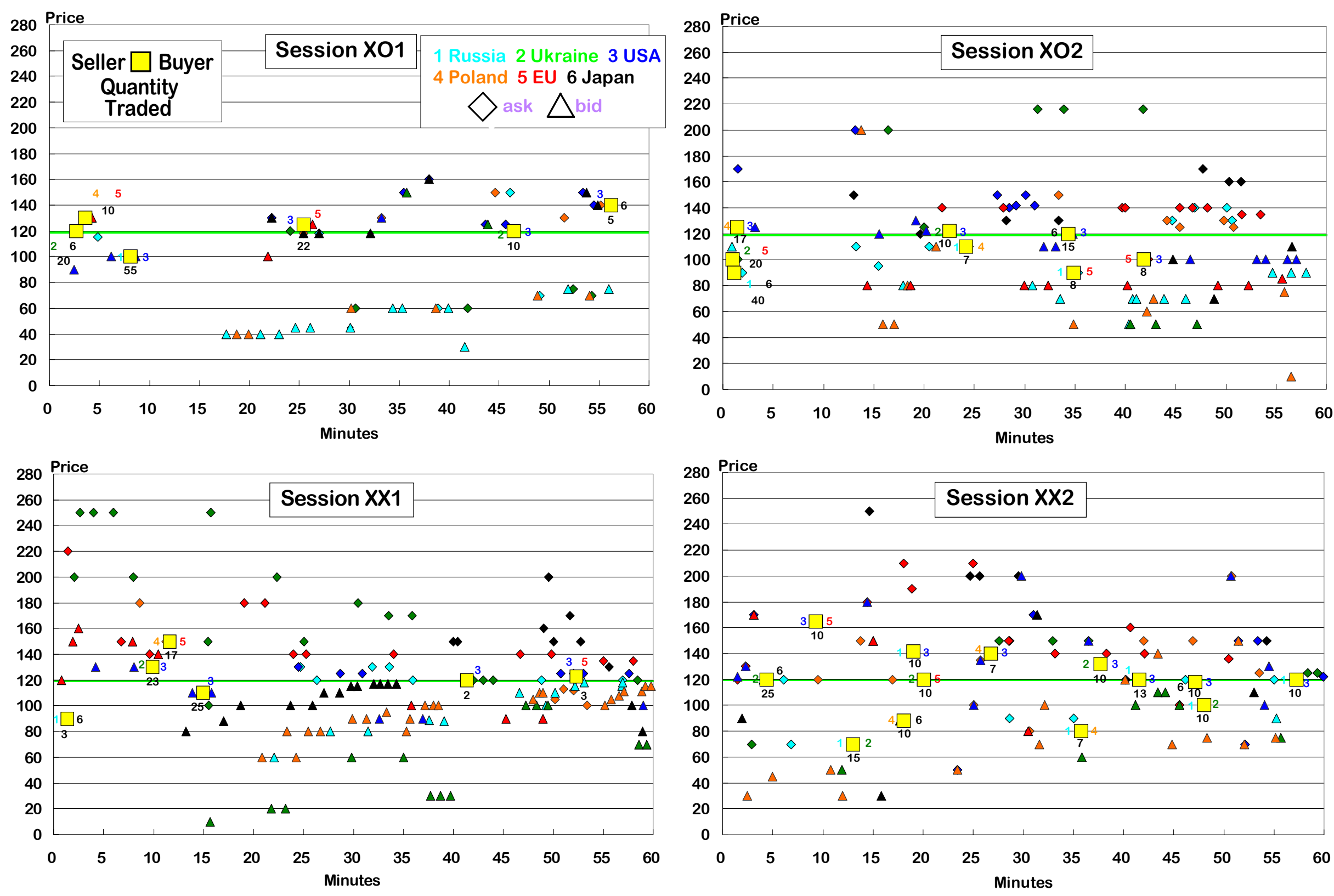

Figure 4-2. Contracts, Asks and Bids in Bilateral Trading 


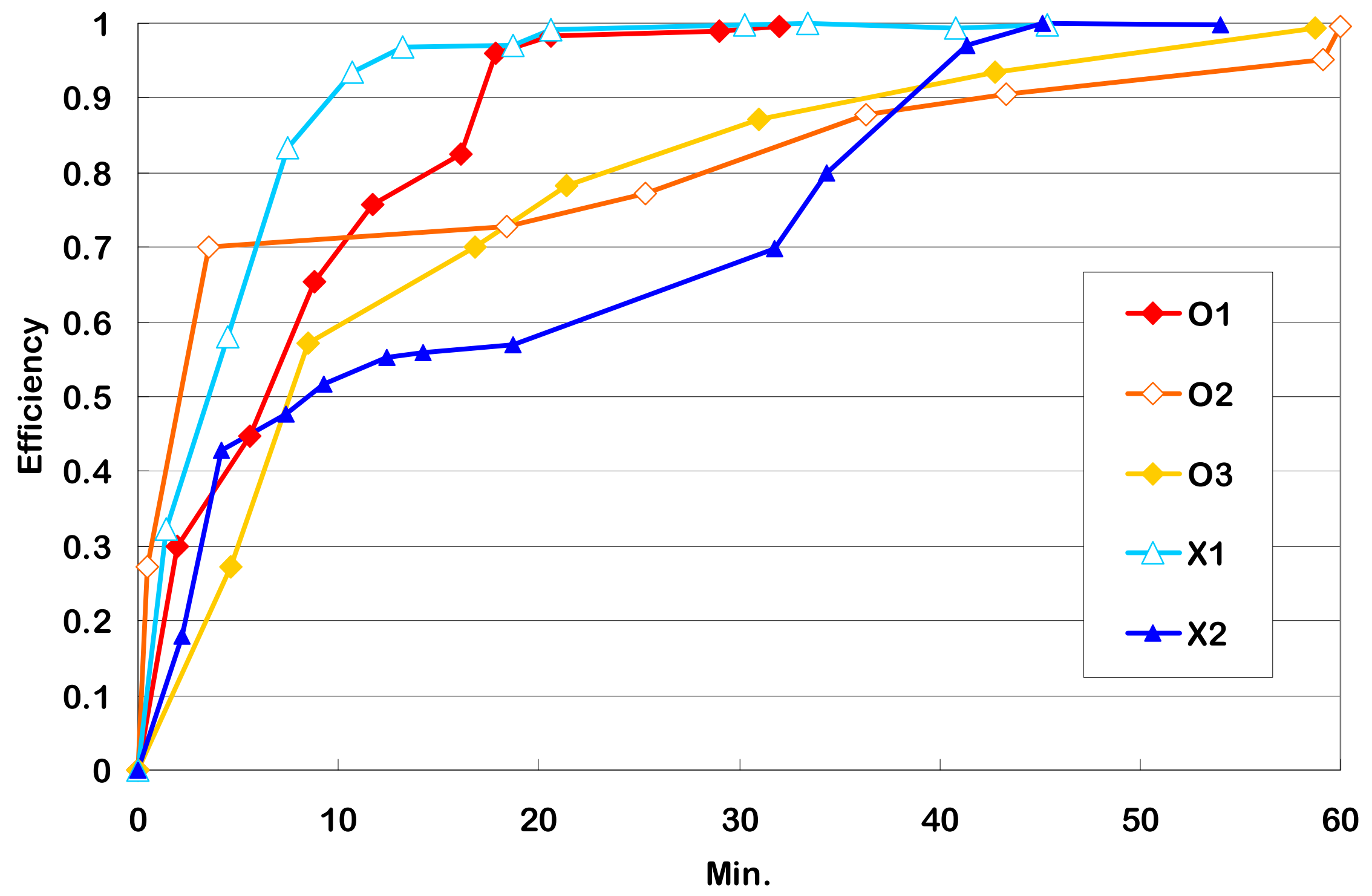

Figure 5. Efficciency over Time in the Double Auction. 

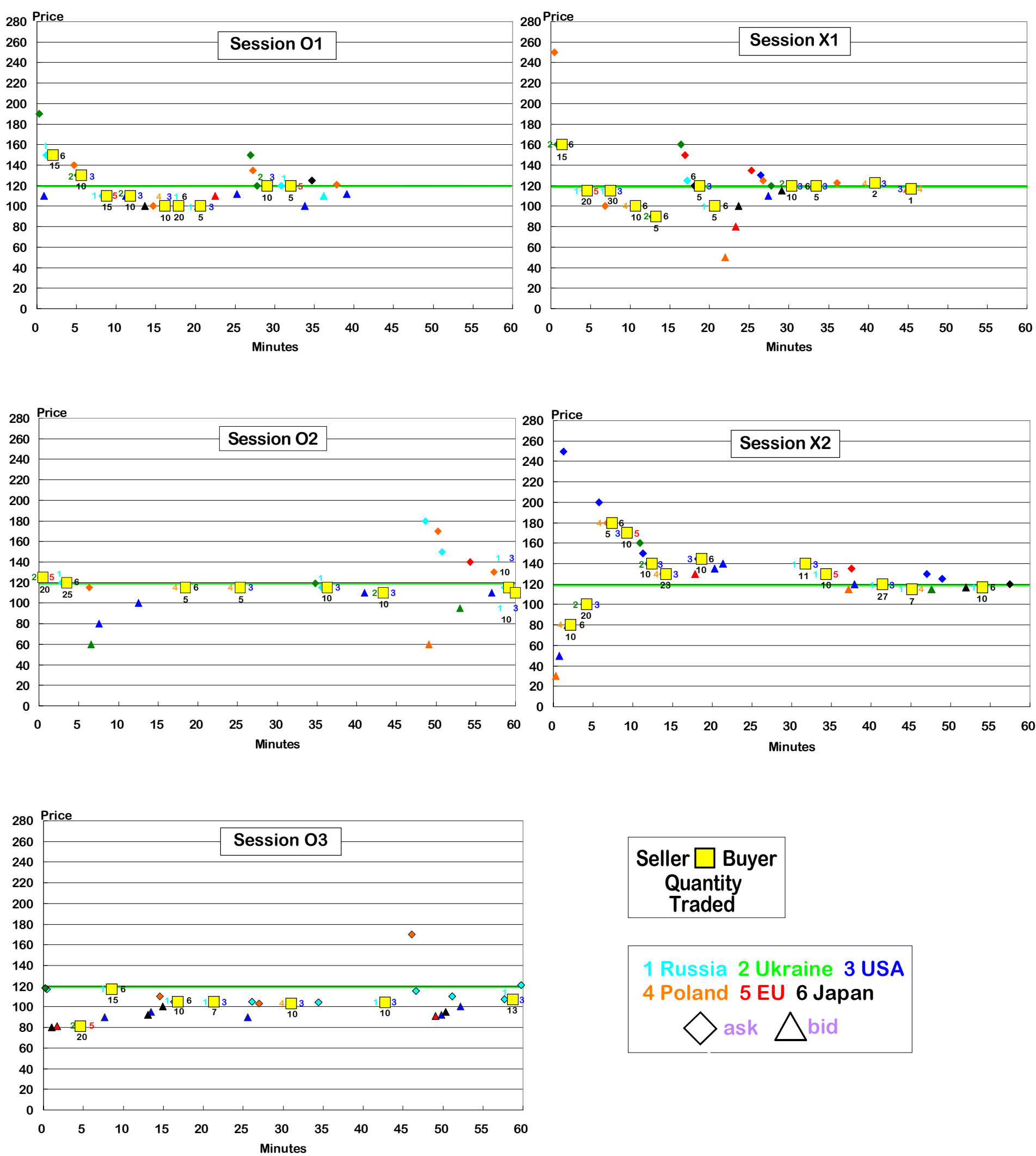

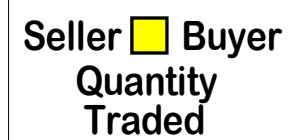

1 Russia 2 Ukraine 3 USA 4 Poland 5 EU 6 Japan

$\diamond$ ask $\triangle$ bid

Figure 6. Contracts, Asks and Bids in the Double Auction 


\section{Price}

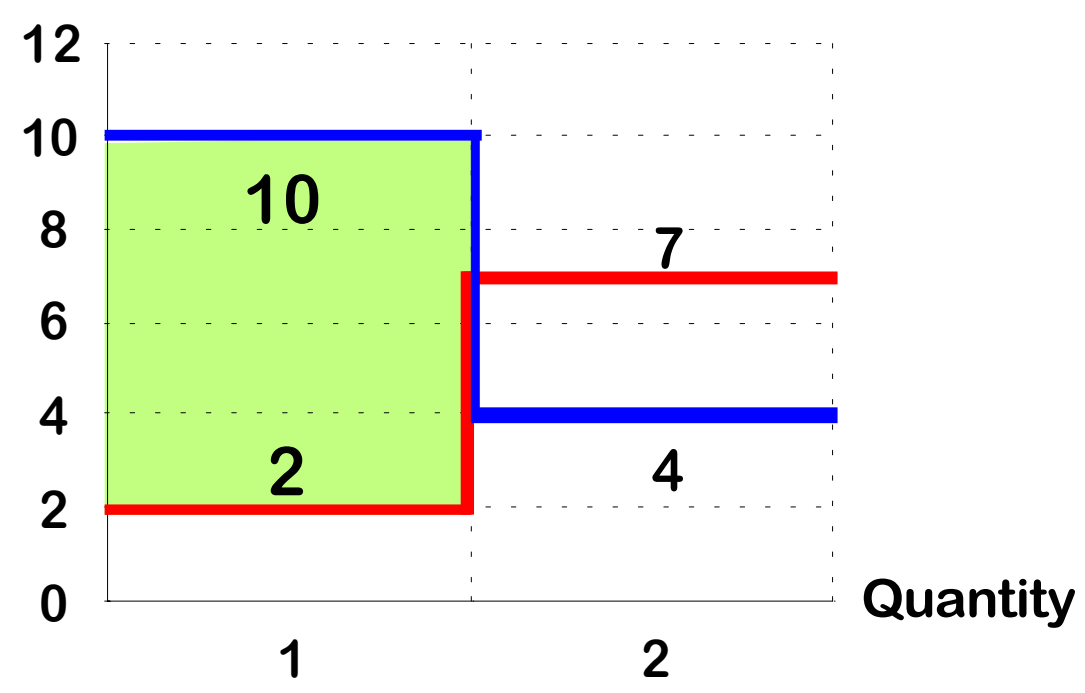

Figure 7. The Dual Role Property 